\title{
Soft-part preservation in a linguliform brachiopod from the lower Cambrian Wulongqing Formation (Guanshan Fauna) of Yunnan, South China
}

Shixue Hu, Zhifei Zhang, Lars E. Holmer, and Christian B. Skovsted

Acta Palaeontologica Polonica 55 (3), 2010: 495-505 doi: http://dx.doi.org/10.4202/app.2009.1106

Linguliform brachiopods were important components of early Cambrian benthic communities. However, exceptionally preserved soft parts in Cambrian linguliform brachiopods are extremely sparse, and the most important findings are from the early Cambrian Chengjiang Konservat Lagerstätte of Kunming, southern China. Here we describe the first record of preserved soft-part anatomy in a linguliform brachiopod from the early Cambrian Guanshan fauna (Wulongqing Formation, Palaeolenus Zone); a unit which is considerably younger than the Chengjiang fauna. The well preserved soft anatomy include linguliform pedicles, marginal setae and, in a few cases, an intact lophophore imprint. The pedicle has pronounced surface annulations, with its proximal-most part enclosing the apex of the ventral pseudointerarea; the pedicle is up to $51 \mathrm{~mm}$ long, corresponding to more than 4 times the sagittal length of the shell, and $12 \%$ of the maximum valve width. In details of their preservation, these new fossils exhibit striking similarities with the linguliforms from the older Chengjiang fauna, and all specimens are preserved in a compressed state as flattened impressions. The new linguliform has an elongate oval to subtriangular shell and an elongate triangular ventral pseudointerarea; the pedicle emerged from an apical foramen through a poorly preserved internal pedicle tube. The new linguliform is most similar to the mostly organic-shelled siphonotretoid-like brachiopod Acanthotretella spinosa, recently described from the classic middle Cambrian Burgess Shale Konservat Lagerstätte, British Columbia, Canada. The new species Acanthotretella decaius sp. nov. is described; it differs from A. spinosa in having a slightly thicker pedicle, and a larger and more rigid, probably partly mineralised shell, indicating that the mostly organic shell of A. spinosa may represent a secondary reduction of shell mineralisation. However, the spine-like setae of the new species are unfortunately poorly preserved only at the margin of the shell, but the new species is referred tentatively to the Superfamily Siphonotretoidea. The occurrence of A. decaius in the Guanshan fauna is the first lower Cambrian (Series 2, early Stage 4) record of both Acanthotretella and siphonotretoids, and it represents the first description of a lophophore and digestive tract from the siphonotretoid lineage.

Key words: Brachiopods, Linguliformea, Konservat Lagerstätte, early Cambrian, China. 
Shixue Hu [hushixue@hotmail.com], Yunnan Geological Survey and Yunnan Institute of Geological Science, No. 87, Dongfeng Lane, East Dongfeng Road, Kunming, 650051, Yunnan Province, China; Zhifei Zhang [elizf@nwu.edu.cn or zhangelle@126.com ], Early Life Institute and Department of Geology, State Key Laboratory for Continental Dynamics, Northwest University, Xi'an, 710069, China (corresponding author). Lars E Holmer [lars.Holmer@pal.uu.se] and Christian B. Skovsted [Christian. Skovsted@geo.uu.se], Department of Earth Sciences, Paleobiology, Uppsala University, Villavägen 16, 75236 Uppsala, Sweden.

This is an open-access article distributed under the terms of the Creative Commons Attribution License (for details please see creativecommons.org), which permits unrestricted use, distribution, and reproduction in any medium, provided the original author and source are credited.

Farif Full text $(1,003.6 \mathrm{kB})$ 